\title{
A Causal Role for Primary Motor Cortex in Perception of Observed Actions
}

\author{
Clare E. Palmer ${ }^{1}$, Karen L. Bunday ${ }^{1}$, Marco Davare ${ }^{1,2}$, and James M. Kilner ${ }^{1}$
}

\begin{abstract}
It has been proposed that motor system activity during action observation may be modulated by the kinematics of observed actions. One purpose of this activity during action observation may be to predict the visual consequence of another person's action based on their movement kinematics. Here, we tested the hypothesis that the primary motor cortex (M1) may have a causal role in inferring information that is present in the kinematics of observed actions. Healthy participants completed
\end{abstract}

\section{INTRODUCTION}

It is now well established that the motor system is active during both action execution and action observation (Gallese, Fadiga, Fogassi, \& Rizzolatti, 1996; di Pellegrino, Fadiga, Fogassi, Gallese, \& Rizzolatti, 1992). A portion of the human ventral premotor cortex (PMv), often thought to be analogous to F5 in the monkey, was the first area in which mirror neurons (neurons that fire during action execution and action observation) were identified; yet it is now clear that populations of neurons throughout the motor system, including PMd and M1, respond to both action execution and observation (Kilner \& Lemon, 2013). However, there is currently a lack of consensus as to the functional role of this motor system activity during action observation. The majority of studies in this field have tested the hypothesis that motor system activity in some way facilitates the perception of the observed action goal (Ferrari, Rozzi, \& Fogassi, 2005; Gallese et al., 1996). More recently, it has been suggested that, as well as a possible role in understanding the goal of an observed action, motor system activity during action observation might enable us to predict the kinematics of the observed action (Kilner, 2011; Kilner, Friston, \& Frith, 2007). Put simply, our ability to infer the goal of an observed action is dependent upon first inferring how that action is performed. There is increasing behavioral evidence from human studies that suggests that we are very sensitive to changes in the kinematics of actions (Ansuini, Cavallo, Bertone, \& Becchio, 2014; Patel, Fleming, \& Kilner, 2012; Alaerts, Senot, et al., 2010; Becchio, Sartori, \& Castiello,

${ }^{1} \mathrm{UCL}$ Institute of Neurology, London, UK, ${ }^{2} \mathrm{KU}$ Leuven an action perception task before and after applying continuous theta burst stimulation (cTBS) over left M1. A neurophysiological marker was used to quantify the extent of M1 disruption following cTBS and stratify our sample a priori to provide an internal control. We found that a disruption to M1 caused a reduction in an individual's sensitivity to interpret the kinematics of observed actions; the magnitude of suppression of motor excitability predicted this change in sensitivity.
2010; Neal \& Kilner, 2010; Sartori, Becchio, Bara, \& Castiello, 2009; Daprati, Wriessnegger, \& Lacquaniti, 2006), and some evidence suggests that activity in the primary motor cortex (M1) is modulated by the kinematics of an observed action (Press, Heyes, \& Kilner, 2011; Alaerts, Swinnen, \& Wenderoth, 2010). This proposed role of the motor system in being sensitive to the kinematics of an observed action is consistent with how neuronal discharge is modulated during action execution (Moran \& Schwartz, 1999; Rizzolatti et al., 1988; Georgopoulos, Schwartz, \& Kettner, 1986). Indeed, the kinematics of executed actions can be decoded from activity in both PMv and M1 (Bansal, Truccolo, Vargas-Irwin, \& Donoghue, 2012).

It has been shown that we are able to infer an individual's subjective state based on the kinematics of their movements (Patel et al., 2012). In this study, observers were able to correctly infer the confidence of participants carrying out a forced-choice discrimination task using only the observed movement kinematics, namely movement speed. A follow-on study (Macerollo, Bose, Ricciardi, Edwards, \& Kilner, 2015) then compared healthy participants and movement disorder patients on the same task. They found that movement disorder patients were significantly worse in their ability to infer confidence from fast movement speeds that differed most from their own. Although this result is consistent with a potential role of action execution networks in inferring information from the kinematics of observed actions, it is unclear whether this behavioral effect is contingent upon activity in the motor cortex.

The aim of this study was to identify a causal role for M1 in this task by disrupting motor excitability using continuous theta burst stimulation (cTBS) over M1. cTBS 
has been traditionally thought to have an inhibitory effect on the output of the targeted area and is therefore used as a "virtual lesion" technique; however, recent studies have shown that this effect is very variable (Hamada, Murase, Hasan, Balaratnam, \& Rothwell, 2013; Huang, Edwards, Rounis, Bhatia, \& Rothwell, 2005). To quantify this intersubject variability, we recorded motor-evoked potentials (MEPs) throughout the task as a neurophysiological marker of the effect of cTBS on corticospinal excitability (CSE). This enabled us, first, to a priori stratify our sample and, second, to correlate changes in CSE with our behavioral effect. We hypothesized that a disruption to M1 would impair an individuals' ability to infer subjective information from the kinematics of observed actions.

\section{METHODS}

\section{Participants}

Twenty-four healthy participants (13 men, 11 women) aged $21-35$ years old (mean $\pm S D: 24.91 \pm 3.83$ ) took part in this study. Participants had no history of neurological or psychiatric illness and had no medical reason to exclude them from having TMS. All participants were right-handed and gave written informed consent before taking part. This study was approved by the UCL research ethics committee, and all testing took place at the UCL Institute of Neurology, Queen's Square.

\section{Experimental Design}

Each participant carried out an action observation behavioral task before and after the application of cTBS. Singlepulse TMS was applied at varying time points throughout the experiment to provide a neurophysiological marker of the effect of cTBS on motor excitability. A baseline measurement of CSE (20 MEPs) was taken before cTBS. Following this, cTBS was applied over left M1 for $40 \mathrm{sec}$. The participants then repeated the action observation task. To measure the effect of cTBS on CSE, 20 MEPs were measured at three time points following CTBS: $10 \mathrm{~min}$ after repetitive stimulation, midway through the behavioral task (approximately 25 min poststimulation), and on completion of the behavioral task (approximately $40 \mathrm{~min}$ poststimulation; see Figure 1A for protocol).

\section{Behavioral Task}

The task was programmed in MATLAB R2013b (The MathWorks, Natick, MA). Participants watched 390 videos divided into two blocks in a pseudorandomized order. In each video, an actor picked up a marble from the center of a table and moved it onto a marker on either the left or the right. The videos were filmed during a previous study in which these participants carried out a two alternate forced-choice discrimination task and were asked to indicate their decision by moving the marble (Patel et al., 2012). In this study, the participants' task was to estimate

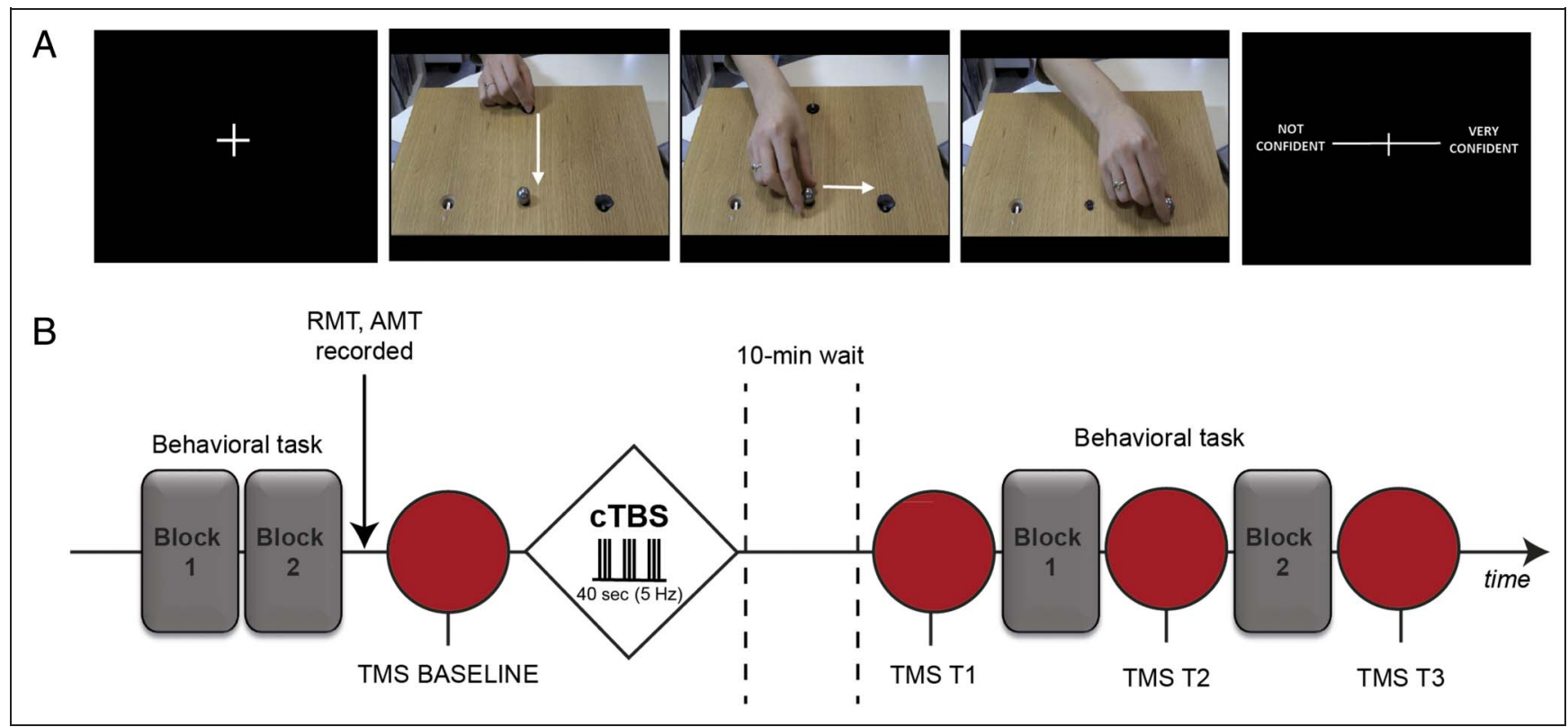

Figure 1. Experimental procedure. (A) Timeline of experimental protocol. The action observation task (gray squares) was completed twice, before and after cTBS. Blocks of 20 MEPs were recorded using single-pulse TMS at baseline and at three time points starting 10 min post-cTBS (red circles). (B) Still frames of the action observation task. Participants $(n=24)$ watched videos in which an individual performed a two alternative forced-choice discrimination task. The left and right sides were assigned to the two choices. The participants in the video had to move the marble to either side to indicate their decision. White arrows indicate hand movement. Movement speed was calculated from the time the hand was released from its starting point (Frame 2) to the time the marble was placed on the left or right of the screen (Frame 4). Observers were instructed to rate the confidence of the participant making the decision in the video after each trial on a scale of 1-100 from "not confident" to "very confident" (Frame 5). 
how confident they thought the actor in the video was in each decision observed. Participants were instructed to rate the confidence of the actor by moving a cursor along a $0-100$ scale bar (Figure 1B). The gender of the actor and the choice location were equally balanced across the videos. Videos were filmed with a bird's eye view of the table so only the actors' hands were visible. All the videos were edited to ensure that the time from the start of the trial to picking up the marble remained the same, and therefore, any difference in RT before picking up the marble could not be used to deduce confidence; the only parameter available to measure confidence was movement speed. Execution time (ET) was used as a proxy for movement speed and was calculated as the time from the moment the marble was removed from its original marker to the time it was placed on one of the choice markers. Catch trials in which the actor hesitated or dropped the marble were removed post hoc. Each participant carried out the task twice before and after cTBS.

\section{Single-pulse TMS}

EMG activity was recorded from the right first dorsal interosseous (FDI) muscle using $\mathrm{Ag} / \mathrm{AgCl}$ cup electrodes in a belly-tendon montage. The EMG signal was amplified $1000 \times$, low-pass filtered at $3 \mathrm{~Hz}$, sampled at $5 \mathrm{kHz}$, and stored for offline analysis (CED 1401 with signal software, version 5.10, Cambridge Electronic Design, Cambridge, UK) A Magstim 200 stimulator (Magstim, Carmarthenshire, UK) was used to deliver monophasic TMS pulses to the hand area of the left primary motor cortex (M1). The figure-of-eight coil ( $9 \mathrm{~cm}$ diameter) was held tangentially to the head over the optimal "hotspot" for producing MEPs. Resting and active motor thresholds (RMT, AMT) were recorded for each participant. Motor threshold was defined as the minimum intensity of the stimulator output, which produced an MEP greater than $50 \mu \mathrm{V}$ on 6 of 10 consecutive pulses. AMT was determined while participants produced a steady isometric contraction against an inert object to produce a constant EMG output at $10 \%$ of their maximum voluntary contraction. The AMT was used to determine the intensity of stimulator output for cTBS; the Magstim Rapid2 (Magstim) was used to find the AMT. Baseline measurements were taken at a stimulator output intensity, which produced a mean MEP amplitude of $\sim 1 \mathrm{mV}$. The same intensity was used throughout the experiment to record MEPs. RMT $=41.76 \pm 7.43 \%$ of maximum stimulator output (MSO; Magstim 200). AMT = $50.28 \pm 7.44 \%$ of MSO (Magstim Rapid2; AMT > RMT due to different stimulators used). Baseline stimulator intensity $=48.16 \pm 9.10 \%$ of MSO (Magstim 200).

\section{Continuous Theta Burst Stimulation}

cTBS was delivered using a Magstim Rapid2 stimulator (Magstim) as a sequence of 200 bursts at a rate of $5 \mathrm{~Hz}$ (total duration $40 \mathrm{sec}$ ). Each burst consisted of three stimuli given at $50 \mathrm{~Hz}$. The stimulator output intensity for cTBS was 80\% of AMT (Huang et al., 2005).

\section{MEP Analysis}

Twenty MEPs were recorded at baseline and then at three time points following cTBS: (1) $10 \mathrm{~min}$ post-cTBS, (2) after Block 1 of the behavioral task, and (3) after Block 2 on completion of the behavioral task. The peak-to-peak amplitude for each individual MEP was measured. MEPs were excluded if there was EMG activity $(>0.1 \mathrm{mV}) 100 \mathrm{msec}$ before the TMS pulse was given (3.85\% of total MEPs). One participant was excluded because of high background EMG activity throughout the baseline resulting in significantly fewer MEPs being analyzed (one-sample $t$ test: $t(23)=55.75, p<.001 ;$ mean difference in number of MEPs at baseline $=5.83$ ). MEPs were logtransformed at the first level to normalize the data and then retransformed at the second level to maintain the original units for MEPs (mV). Magnitude of MEP suppression was calculated as the difference between the average normalized MEP amplitude at baseline and the grand average of normalized MEP amplitude at three time points post-cTBS. MEP amplitude was stable across time points poststimulation: A repeated-measures ANOVA revealed no significant main effect of Time, and none of the pairwise comparisons were significant $(p>.1)$. An inhibitory response to cTBS was defined as a magnitude of MEP suppression greater than 0 and a facilitatory response less than 0 (López-Alonso, Cheeran, Río-Rodríguez, \& Fernándezdel-Olmo, 2014; Hamada et al., 2013). Baseline MEP amplitudes were also compared between groups using a two-tailed independent samples $t$ test to ensure that there was no baseline difference between groups.

The stability of MEP amplitudes across the three time points post-cTBS was determined using a one way repeated-measures ANOVA. To see if the MEP values were reliable as well as stable over time post-cTBS, a correlation analysis between the average MEP amplitudes for each participant at each time point was conducted for all pairwise combinations of time point (e.g., T1 vs. T2, T2 vs. T3, T1 vs. T3). To determine whether MEP amplitudes were consistently decreased or increased within participants dependent on their overall categorization into either the inhibitory or facilitatory groups, nonparametric sign tests were conducted between the difference in MEP amplitude from baseline at each time point and the grand average difference in MEP amplitude from baseline (used to categorize participants).

\section{Behavioral Data Analysis}

Confidence ratings were ordered based on ET from fastest to slowest and grouped into 10 bins per participant. ET bins were divided to have as close to equal numbers as possible. The first block, which had a total of 190 videos, had 19 in each bin, and the second block with 
192 videos had 19 in Bins 1-9 and 21 in the last bin. The range of ETs in each bin were as follows: $863-1023 \mathrm{msec}$ (Bin 1); 1023-1064 msec (Bin 2); 1068-1103 msec (Bin 3); 1103-1128 msec (Bin 4); 1129-1154 msec (Bin 5); 1154$1178 \mathrm{msec}$ (Bin 6); 1178-1223 msec (Bin 7); 1224-1259 msec (Bin 8); 1269-1325 msec (Bin 9); 1329-1655 msec (Bin 10). The mean confidence rating of each bin was plotted against ET. The gradient of this line was used as a measure of sensitivity to movement speed. The mean confidence rating across conditions for each participant was deducted from the mean confidence rating at each bin for each condition for each participant to normalize the scores and remove any between-subject variance in use of the confidence scale.

The outcome measure "change in gradient" (difference in gradient [sensitivity] before and after cTBS) was analyzed between participants (inhibition and facilitation groups based on change in mean MEP amplitude postcTBS) using a two-tailed independent samples $t$ test. Post hoc tests were then carried out to provide more details about the specificities of this effect. One-sample $t$ tests were conducted to identify whether the change in gradient for each group was significantly different from zero. Nonparametric permutation tests were also conducted to corroborate the findings from the parametric tests using a statistical test that makes no assumption as to the underlying distribution of the observed data. Here, for the change in gradient data, the condition labels (inhibition group or facilitation group) were randomly permuted and the group mean difference calculated 1,000,000 times. Only unique group means were selected in ensuring that the permuted distribution was not biased. The distribution of mean differences was calculated, and the position of the true mean difference was determined to identify if the difference between the groups was significant. The same analysis was conducted separately for the inhibition and facilitation groups using the pre- and post-cTBS data points to determine in each group if the change in gradient observed was significantly increased or decreased from zero. Baseline sensitivity to observed movement speed before cTBS was also compared between groups using a two-tailed independent samples $t$ test to ensure that there was no baseline difference between groups.

A linear regression analysis was used to determine the predictive relationship between (1) "observed movement speed" and "inferred confidence ratings," and (2) "change in mean MEP amplitude" and "change in gradient." Although there are alternative predictions one could make regarding the shape of these relationships, based on previous literature (Patel et al., 2012), for the purpose of this study these relationships are assumed to be linear.

The response to indicate confidence required a motor action with higher confidence ratings requiring a greater number of key presses along the 0-100 scale bar. To ensure that any changes in sensitivity to movement speeds were not caused simply by an impairment at the motor level reducing the overall number of key presses produced, mean confidence ratings in the fastest three time bins (highest confidence ratings) were compared before and after cTBS using paired sample $t$ tests for the inhibition group. For all outcome measures, assumption of a normal distribution (using the Shapiro-Wilk test of normality) was verified.

\section{RESULTS}

\section{Effect of cTBS on CSE}

MEPs recorded from the right FDI of each participant before and after cTBS provided a physiological measure of the efficacy of cTBS in disrupting the motor cortex. The change in cortical excitability following cTBS was highly variable between participants and over time in an individual participant. Representative MEP waveforms averaged over 20 MEPs (baseline) or the grand average $(3 \times 20$ MEPs) post-cTBS are shown from two individuals, which had differential responses to cTBS (Figure 2A, 2B). Of 24 participants, 15 showed post-cTBS inhibition (mean \pm $S D$ : $37.2 \pm 21.4 \%$ decrease) and 9 showed post-cTBS facilitation ( $42.8 \pm 29.6 \%$ increase; Figure $2 \mathrm{C})$ defined by a positive or negative change in the grand average postcTBS MEP amplitude from baseline (see Methods for more details on categorization). There were no significant differences between the two groups in baseline MEP amplitude, $t(22)=-0.817, p=.423$ (mean difference in MEP amplitude: $0.15 \pm 0.09 \mathrm{mV}$ ) before cTBS.

To ensure MEP amplitudes were stable across time points poststimulation, a one-way repeated-measures ANOVA was conducted; this revealed no significant main effect of Time, and none of the pairwise comparisons were significant $(p>.1)$. Correlation analyses, conducted to determine the reliability of the MEP amplitudes over time post-cTBS, revealed significant positive relationships between average MEP amplitudes at all time points (T1 vs. T2: $r=.71, p<.001$; T2 vs. T3: $r=.54, p=.018$; T1 vs. T3: $r=.65, p<.001)$. Moreover, sign tests conducted to determine whether MEP amplitudes were consistently decreased or increased, dependent on their overall categorization into either the inhibition or facilitation groups, were all significant (T1: $p=.0075 ; \mathrm{T} 2: p=.0034 ; \mathrm{T} 3: p=$ .0063 ), which demonstrates that the sign of the difference between the average MEP amplitude at each time point and the baseline MEP amplitude was consistently the same as the sign associated with the group into which participants were categorized.

\section{Effect of cTBS on Sensitivity to Action Kinematics}

ETs from all the videos shown were divided into 10 bins, and the mean confidence rating across all participants for each bin was plotted before and after cTBS (see Figure 3). In all cases, movement speed significantly predicted inferred confidence ratings, $r^{2}=.98, F(1,237)=472.37, p<.001$; as observed movement speed increased, participants' 
Figure 2. Changes in CSE following cTBS. MEPs recorded from the right FDI muscle before and after stimulation. Mean baseline (blue) MEP waveform averaged over 20 MEPs for each participant. Post-cTBS (red = inhibition; green $=$ facilitation) MEP waveforms averaged across three time points following cTBS ( $n=60$ MEPs).

(A) Representative mean (SEM $=$ shaded area) MEP waveform before stimulation (blue) and after stimulation (red) from a participant in the inhibition group.

(B) Representative mean (SEM = shaded area) MEP waveform before stimulation (blue) and after stimulation (green) from a participant in the facilitation group. (C) Mean (SEM) percentage change in MEP amplitude for the inhibition group (red) and the facilitation group (green). $\mathrm{S}=$ stimulus artifact.

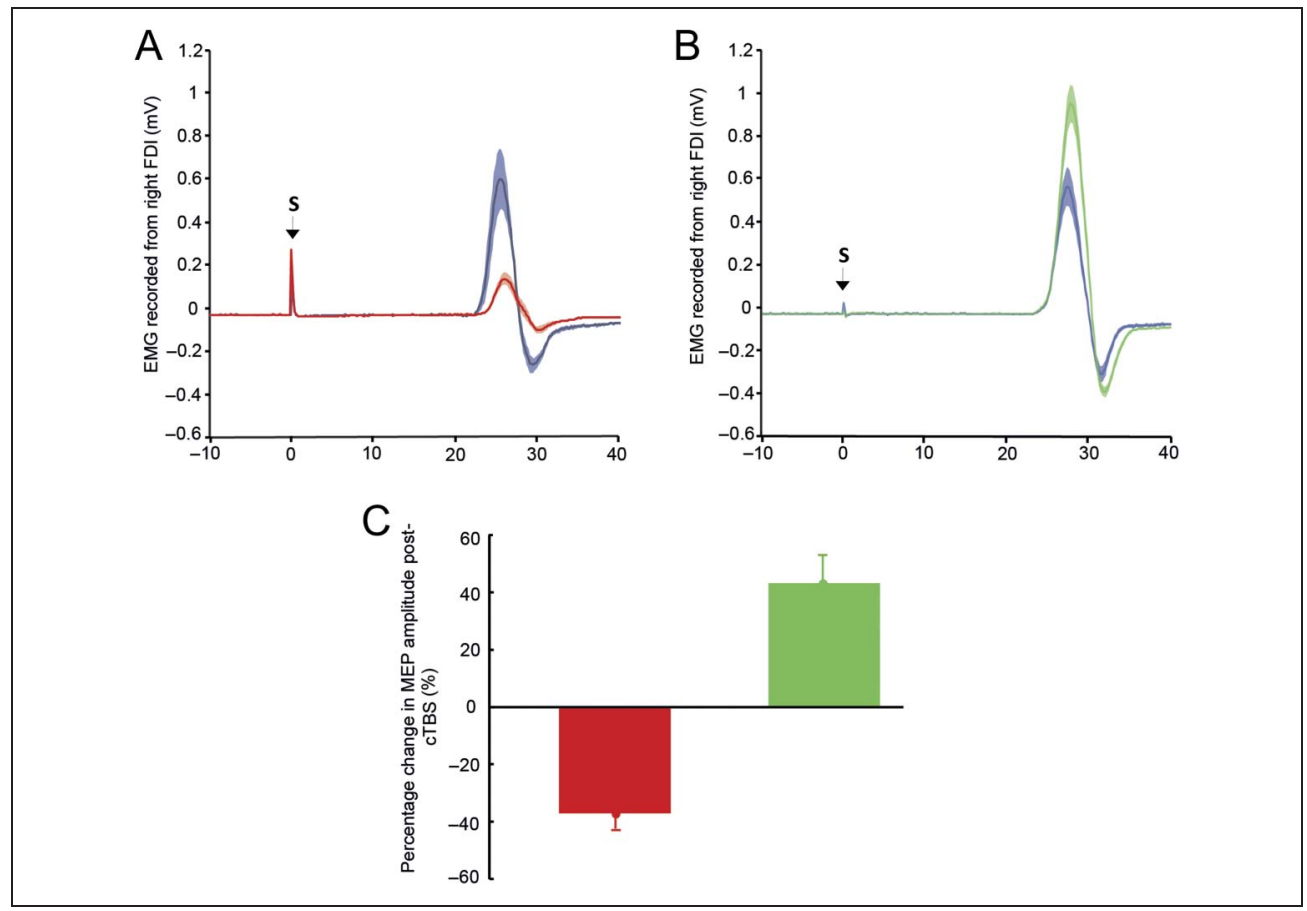

Figure 3. cTBS reduces sensitivity to observed kinematic information in inhibition group. Mean confidence ratings for observed ETs were divided into 10 bins before cTBS (solid line) and after cTBS (dashed line).

Movement speed significantly predicted inferred confidence ratings for all graphs, $r^{2}=.98$, $F(1,237)=472.37, p<.001$. (A) Mean (SEM) confidence ratings for observed ETs for the inhibition group only before and after cTBS. Significant change in gradient (measure of sensitivity) before and after cTBS, $t(14)=-2.25$, $p=.041$ (mean $\pm S D$ change in gradient $=-0.0095 \pm$ 0.016). (B) Mean (SEM) confidence ratings for observed ETs for the facilitation group only before and after cTBS. No significant change in gradient (measure of sensitivity) before and after cTBS, $t(8)=0.92$, $p=.39$ (mean $\pm S D$ change in gradient $=-0.0041 \pm 0.013)$.

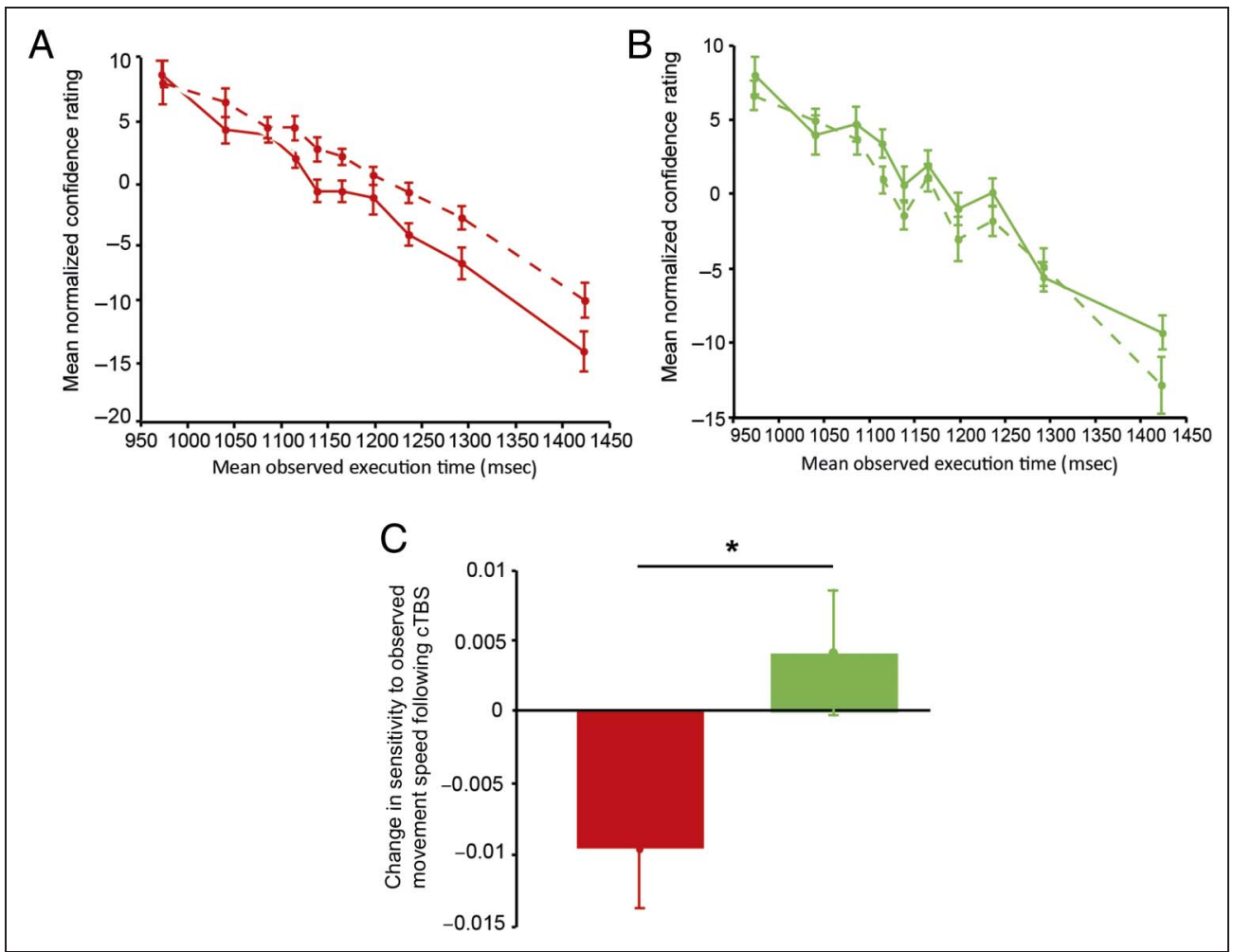

(C) Change in gradient (sensitivity) following cTBS for the inhibition group (red) and facilitation group (green). There was a significant between-subject difference in change in sensitivity, $t(22)=-2.1, p=.047$. The facilitation group did not show a significant change in sensitivity from $0, t(8)=0.92, p=.39$ (mean $\pm S D$ change in gradient $=0.0041 \pm 0.013$ ) 
confidence ratings increased replicating previous findings (Macerollo et al., 2015; Patel et al., 2012). The gradient of this regression was used as a measure of sensitivity in this study. A participants' sensitivity to movement speed was thus defined as the rate of change in confidence over unit change in movement speed with the assumption that a shallower gradient represented a decrease in sensitivity.

As noted previously, the effects of cTBS over M1 are highly variable across individuals. By recording MEPs throughout the task, we were able to stratify our sample based on the effect of cTBS on CSE and correlate this with changes in behavior. Participants were divided into an inhibition or facilitation group based on a positive or negative change in mean MEP amplitude following cTBS. There was a significant difference in the change in gradient (sensitivity) following cTBS between the inhibition and the facilitation groups, $t(22)=-2.1, p=.047$. Post hoc $t$ tests demonstrated that the inhibition group showed a significant reduction in sensitivity to observed movement speed following cTBS, $t(14)=-2.25, p=$ .041 (mean change in gradient $=-0.0095 \pm 0.016$; Figure 3B; not corrected for multiple comparisons), whereas the facilitation group showed a slight but nonsignificant increase in sensitivity, $t(8)=0.92, p=.39$ (mean change in gradient $=0.0041 \pm 0.013$ ). There were no significant differences between the two groups in the baseline measures of sensitivity to observed movement speed, $t(22)=-1.492, p=.150$ (mean difference in gradient: $-0.011 \pm 0.006$ ) before cTBS.

In addition, nonparametric permutation tests, which make no assumption as the distribution of the underlying data, were used to corroborate the above findings. The permutation test revealed a significant group difference in change in gradient following cTBS between the inhibition and facilitation groups $(p=.018)$. Moreover, the same test was conducted separately for each group to determine if the difference in sensitivity to movement was significantly increased or decreased following cTBS. The analysis revealed that the true mean difference was significantly less than zero for the inhibition group $(p=.011)$ and not significantly different from zero for the facilitation group ( $p=.168$ ). This supports our original finding that sensitivity to observed movements is significantly reduced from zero in the inhibition group $(p<.05$ corrected for multiple comparisons).

Here, it is clear that the nonparametric tests were more sensitive at detecting significant differences in the behavioral data, which suggests that there may be no clear bimodality in the observed MEP data. Indeed, a regression analysis conducted across all of the data revealed a significant correlation between the change in mean MEP amplitude and a change in sensitivity across all participants, such that change in MEP amplitude significantly predicted the change in sensitivity to observed movements (parametric: $r^{2}=.21, r=.47, p=.022$; nonparametric: $r_{\mathrm{s}}=.48, p=.019$; Figure 4 ).
To ensure that the reduction in sensitivity to movement speed in the inhibition group was not simply caused by a motor impairment leading to a reduction in the number of key presses needed to produce the highest confidence ratings, mean confidence ratings at each movement speed time bin for the three fastest bins (which required the greatest number of key presses) were compared before and after cTBS for the inhibition group using paired sample $t$ tests, and no significant differences were found $(p>5)$. It is clear that the decrease in sensitivity to movement speed after cTBS was not caused by a reduction in the participants' own movements. In fact, the overall mean confidence rating after cTBS for the inhibition group significantly increased, demonstrating that overall these participants generated a greater number of key presses $(t(13)=-2.811, p=$ .015). There is a greater cluster of responses at the upper end of the confidence scale for these participants, as they are less sensitive to different observed movement speeds and therefore rate them all as more similar via the proxy that we have used in this experiment, confidence; this is what underlies the decrease in gradient following cTBS in the inhibition group.

\section{DISCUSSION}

Here we have shown that a disruption to M1 excitation following the application of cTBS altered an individuals' sensitivity to infer information from the kinematics of an observed action. Participants were divided into two subgroups based on a decrease or increase in M1 excitation post-cTBS. We found a significant difference in behavior between the two groups as well as a correlation between the magnitude of change in MEP amplitude and the

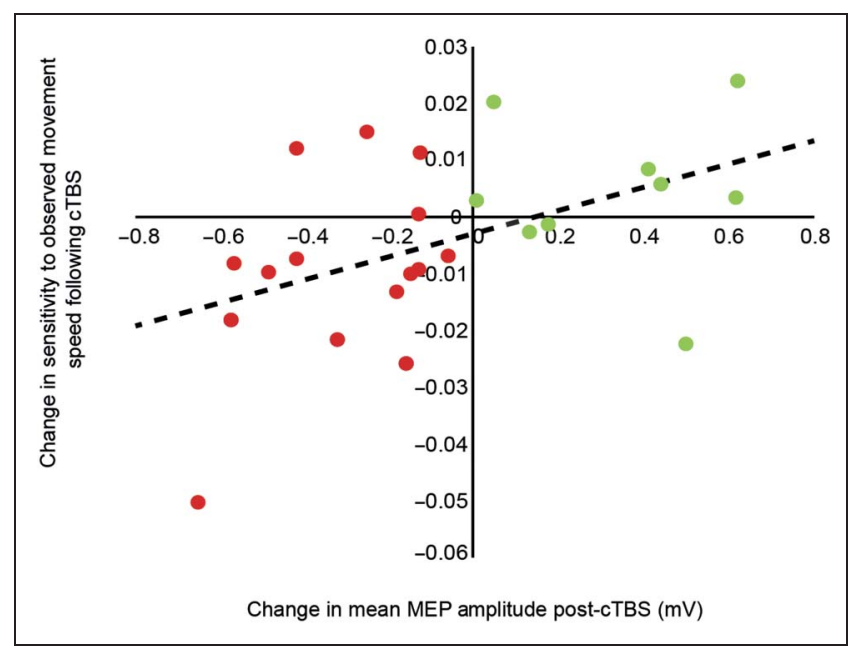

Figure 4. Magnitude of MEP suppression predicts change in sensitivity to movement speed following cTBS. A linear regression analysis demonstrates a significant predictive relationship between the mean change in MEP amplitude and change in sensitivity (gradient) following CTBS (parametric: $r^{2}=.21, p=.023$; nonparametric: $r_{\mathrm{s}}=-.048$, $p=.019)$. Facilitation group: green; inhibition group: red. 
degree of change in sensitivity to movement speed. This result demonstrates, first, the variability in the cortical response to cTBS and, second, the importance of having an independent physiological measure that can explain some of this variability.

Previous studies have demonstrated large interindividual variability in response to TMS protocols that test synaptic plasticity in the human motor system (López-Alonso et al., 2014; Hamada et al., 2013; Ridding \& Ziemann, 2010). In fact, Hamada et al. (2013) systematically investigated the variability of cTBS effects in a large subject sample and found that only $42 \%$ of participants showed the expected inhibitory response whereas the remaining $58 \%$ surprisingly showed a facilitatory response (Hamada et al., 2013). Despite this, many "virtual lesion" studies do not use a neurophysiological marker to quantify whether the disruptive TMS protocol used has efficiently affected the ROI. In this study to account for this variability, MEPs were recorded from the hand before and after cTBS, as in Hamada et al.'s (2013) study, to provide one measure of the extent to which the motor cortex was disrupted by the repetitive stimulation. MEP amplitude was averaged over three time points following cTBS to provide the best estimate of the change in CSE caused by the cTBS over time. It is still unclear how cTBS interacts with motor cortex activity and CSE; however, in this study MEP amplitude provided a readily quantifiable measure of one effect of cTBS on CSE, which can be used to infer levels of motor cortex activity. In addition, it is important to stress that in this study the MEPs were recorded when the participants were at rest and not when they were performing the observation task. Therefore, any differences in the MEP cannot be attributed to modulations in the motor system during action observation. The magnitude of change in cortical excitation, measured by MEP amplitude, predicted an individuals' sensitivity to the kinematics of observed actions. This provides further support for the individual variability in response to cTBS and the importance of quantifying the effect of the stimulation to avoid Type 2 errors. Cortical excitability is much harder to quantify outside the motor system; therefore, it is essential that reliable behavioral tests for other ROIs are developed as a readout of the efficiency of repetitive TMS protocols.

The vast majority of virtual lesion studies using repetitive stimulation typically include a sham control group to determine the specificity of the ROI in causally influencing any change in behavior recorded. One caveat of sham controls is that they either involve no stimulation or stimulation to another area, which is fundamentally different from the real stimulation condition. In the current study, a positive or negative change in MEP amplitude following cTBS was used to categorize participants into a facilitation or inhibition group. This between-subject categorization provided the optimal internal control for our study and negated the requirement for a control sham condition. Here the facilitation and inhibition groups received the same type and duration of cTBS stimulation; there- fore, any differences in behavior between these groups can be attributed to the orthogonal measure of MEP modulation. This also removes any potential order confounds. All participants completed the action observation task twice in the same order, before and after cTBS; therefore, it could be argued that any changes in performance post-cTBS are due to boredom or lack of attention. However, as we only see a decrease in sensitivity in a subset of the sample who show a negative change in MEP amplitude, it is unlikely that this can explain the data.

Despite the facilitation group showing an almost equal mean increase in MEP amplitude as the inhibition group showed decrease, the "facilitators" did not show a significant behavioral difference in sensitivity to observed kinematic information before and after cTBS, $t(8)=0.92, p=$ .39 (mean gradient before cTBS: $-0.038 \pm 0.014$; mean gradient after cTBS: $-0.042 \pm 0.019)$. However, a significant regression between magnitude of MEP suppression and change in sensitivity supports a shift in behavior in the facilitation group in the appropriate direction. One explanation for this may be that the facilitation group had too few participants to reach significance. Alternatively, this dichotomy may be due to the mechanisms underlying cTBS and the specific populations of interneurons being activated. The recruitment of early and late I-waves has been highlighted as a key indicator of whether an individual will show an inhibitory or facilitatory response to TBS protocols (Hamada et al., 2013), which suggests that differential circuits may be activated in the inhibition and facilitation groups. Moreover, an inhibitory effect on CSE following cTBS has been associated with an increase in spontaneous beta oscillatory activity over M1 and subsequent increase in RT in a cued finger-tapping task; however, those showing facilitation of CSE displayed a decrease in RT, but no significant change in spontaneous beta power (McAllister et al., 2013). Inhibition of neurons, which causes a decrease in CSE, may have a larger overall effect at promoting high-frequency synchronous network activity than activation of neurons, which cause an increase in CSE. Differences in synchronous activity over M1 following cTBS may therefore provide a more optimal method of categorizing participants and accounting for the differential behavior seen here than CSE alone.

This study provides evidence of a causal role of the primary motor cortex in action perception. Initial studies of mirror neurons (Gallese et al., 1996; di Pellegrino et al., 1992) did not find any evidence of these neurons in M1, and it was therefore assumed that M1 had no role at all in action perception. Subsequent to this, three studies have reported modulation of neuronal activity in M1 during action observation (Vigneswaran, Philipp, Lemon, \& Kraskov, 2013; Dushanova \& Donoghue, 2010; Tkach, Reimer, \& Hatsopoulos, 2007). In addition, it is well established that MEPs in hand muscles resulting from single-pulse TMS of M1 are facilitated during action observation tasks in a muscle-specific manner (Fadiga, 
Fogassi, Pavesi, \& Rizzolatti, 1995) and beta power modulations during action observation are known to have their source in M1 (Koelewijn, van Schie, Bekkering, Oostenveld, \& Jensen, 2008). However, the functional role of this activity remained unclear. The results of our study provide evidence that M1 is functionally employed during action observation and that activity in M1 during action observation is related to the kinematics of an observed action. This finding would be consistent with the predictive account of motor system activation during action observation, which posits that mirror neurons fire as part of a generative model that predicts the sensory consequences of an action. Within this model, the motor system is active during action observation, as it is the best model to generate a prediction of how the observed hand will move through space: the kinematics of the observed action. According to this account, disruption to the motor system during action observation should disrupt an individuals' ability to form an accurate prediction of the kinematics of an observed action. The results of this study support this view; however, it must be emphasized that this study was designed to explore the role of M1 in action observation and not to specifically identify if M1 plays a predictive or reactive mechanism in action observation. Future work is necessary to tease apart the mechanistic role of M1 within this predictive coding account.

There have been a number of studies that have used "virtual lesion" techniques to identify causal roles of different areas of the motor system in action observation (for a review, see Avenanti, Candidi, \& Urgesi, 2013). In particular, the inferior frontal cortex has been implicated in integrating the spatial and temporal features of observed kinematics (Tidoni, Borgomaneri, di Pellegrino, \& Avenanti, 2013; Pobric \& Hamilton, 2006), and the dorsal premotor cortex appears to play a role in correctly predicting the temporal consequences of an occluded observed action (Stadler et al., 2012). However, within this literature, no study has been reported that has examined the necessary role for the primary motor cortex in inferring information from observed kinematics, which is the focus of this study. In addition, importantly, these brain stimulation studies lack an orthogonal marker of cortical excitability to identify the extent of disruption caused by the repetitive lesioning technique, an innovative and highly necessary method that we have employed here.

However, although we know that M1 function was disrupted in this study through the subsequent modulation of the MEP, it is possible that the perceptual change reported here could be due to disruption in areas with dense connectivity with M1. During action execution it has been suggested that neurons within the PMv encode the direction of an action in space necessary to acquire a specific target (goal); this information is then transmitted to M1 and combined with muscle and joint information to determine how that action would be carried out (kinematics). This putative gradient of movement processing may be mirrored within the action observation network, suggesting a strong reliance of M1 on PMv for kinematic processing. The precise nature of the interplay between PMv and M1 during action observation should form the basis of future studies; for example, paired-pulse conditioning can be used to determine the magnitude of disruption in the PMv in response to cTBS over M1 to confirm these conclusions.

Here we have shown an effect of cTBS of M1 on action perception; this could either be due to a specific disruption to the motor system or nonspecific effect in perceptual or decision-making domains. There is compelling evidence that the effects seen here are indeed due to disruption of processes in M1. A previous study using the same stimuli (Macerollo et al., 2015) found the same decrease in sensitivity to kinematic information that we found in the inhibition group in a sample of patients with movement disorders compared with healthy age-matched controls. In this previous study, the participants were asked to complete a control task in which participants were asked to judge the time when a target traveling at a constant speed would reappear after disappearing behind an occluder. There was no significant difference in performance between the patient and control groups on this task, suggesting that the behavioral effect seen in the action observation task was specifically due to a disruption of the participant's own kinematic motor representation rather than motion perception in general. Despite the absence of a similar control task in the current study, using this information it can be inferred that the worsening in behavior seen in the inhibition group was due to a similar specific disruption of the motor system caused by rTMS. Future studies could aim to determine the specificity for M1 in the processing of kinematic actions.

In summary, this is a novel study demonstrating for the first time that M1 has a causal role in the perception of kinematic information from observed actions. It sheds new light in defining the role of M1 in action perception and within the interconnected "mirror neuron network."

\section{Acknowledgments}

C. E. P. is funded by a Wellcome Trust PhD studentship and is in the 4-year PhD Programme in Neuroscience at UCL. M. D. is funded by a BBSRC David Phillips fellowship (UK), the Royal Society (UK), and an FWO Odysseus project (Fonds Wetenschappelijk Onderzoek, Belgium).

Reprint requests should be sent to Clare E. Palmer, Sobell Department for Motor Neuroscience and Movement Disorders, Institute of Neurology, University College London (UCL), 33 Queen Square, London, United Kingdom, WC1N 3BG, or via e-mail: clare.palmer.13@ucl.ac.uk.

\section{REFERENCES}

Alaerts, K., Senot, P., Swinnen, S. P., Craighero, L., Wenderoth, N., \& Fadiga, L. (2010). Force requirements of observed object lifting are encoded by the observer's motor system: A TMS study. European Journal of Neuroscience, 31, 1144-1153. 
Alaerts, K., Swinnen, S. P., \& Wenderoth, N. (2010). Observing how others lift light or heavy objects: Which visual cues mediate the encoding of muscular force in the primary motor cortex? Neuropsychologia, 48, 2082-2090.

Ansuini, C., Cavallo, A., Bertone, C., \& Becchio, C. (2014). The visible face of intention: Why kinematics matters. Frontiers in Psychology, 5, 815.

Avenanti, A., Candidi, M., \& Urgesi, C. (2013). Vicarious motor activation during action perception: Beyond correlational evidence. Frontiers in Human Neuroscience, 7, 185.

Bansal, A. K., Truccolo, W., Vargas-Irwin, C. E., \& Donoghue, J. P. (2012). Decoding 3D reach and grasp from hybrid signals in motor and premotor cortices: Spikes, multiunit activity, and local field potentials. Journal of Neurophysiology, 107, 1337-1355.

Becchio, C., Sartori, L., \& Castiello, U. (2010). Toward you: The social side of actions. Current Directions in Psychological Science, 19, 183-188.

Daprati, E., Wriessnegger, S., \& Lacquaniti, F. (2006). Kinematic cues and recognition of self-generated actions. Experimental Brain Research, 177, 31-44.

di Pellegrino, G., Fadiga, L., Fogassi, L., Gallese, V., \& Rizzolatti, G (1992). Understanding motor events: A neurophysiological study. Experimental Brain Research, 91, 176-180.

Dushanova, J., \& Donoghue, J. (2010). Neurons in primary motor cortex engaged during action observation. European Journal of Neuroscience, 31, 386-398.

Fadiga, L., Fogassi, L., Pavesi, G., \& Rizzolatti, G. (1995). Motor facilitation during action observation: A magnetic stimulation study. Journal of Neurophysiology, 73, 2608-2611.

Ferrari, P. F., Rozzi, S., \& Fogassi, L. (2005). Mirror neurons responding to observation of actions made with tools in monkey ventral premotor cortex. Journal of Cognitive Neuroscience, 17, 212-226.

Gallese, V., Fadiga, L., Fogassi, L., \& Rizzolatti, G. (1996). Action recognition in the premotor cortex. Brain, 119, 593-609.

Georgopoulos, A. P., Schwartz, A. B., \& Kettner, R. E. (1986). Neuronal population coding of movement direction. Science (New York, N.Y.), 233, 1416-1419.

Hamada, M., Murase, N., Hasan, A., Balaratnam, M., \& Rothwell, J. C. (2013). The role of interneuron networks in driving human motor cortical plasticity. Cerebral Cortex, 23, 1593-1605.

Huang, Y.-Z., Edwards, M. J., Rounis, E., Bhatia, K. P., \& Rothwell, J. C. (2005). Theta burst stimulation of the human motor cortex. Neuron, 45, 201-206.

Kilner, J. M. (2011). More than one pathway to action understanding. Trends in Cognitive Sciences, 15, 352-357.

Kilner, J. M., Friston, K. J., \& Frith, C. D. (2007). Predictive coding: An account of the mirror neuron system. Cognitive Processing, 8, 159-166.

Kilner, J. M., \& Lemon, R. N. (2013). What we know currently about mirror neurons. Current Biology, 23, R1057-R1062.

Koelewijn, T., van Schie, H. T., Bekkering, H., Oostenveld, R., \& Jensen, O. (2008). Motor-cortical beta oscillations are modulated by correctness of observed action. Neuroimage, 40, 767-775.

López-Alonso, V., Cheeran, B., Río-Rodríguez, D., \& Fernándezdel-Olmo, M. (2014). Interindividual variability in response to non-invasive brain stimulation paradigms. Brain Stimulation, 7, 372-380.

Macerollo, A., Bose, S., Ricciardi, L., Edwards, M. J., \& Kilner, J. M. (2015). Linking differences in action perception with differences in action execution. Social Cognitive and Affective Neuroscience, 10, 1121-1127.

McAllister, C. J., Rönnqvist, K. C., Stanford, I. M., Woodhall, G. L., Furlong, P. L., \& Hall, S. D. (2013). Oscillatory beta activity mediates neuroplastic effects of motor cortex stimulation in humans. Journal of Neuroscience, 33, 7919-7927.

Moran, D. W., \& Schwartz, A. B. (1999). Motor cortical representation of speed and direction during reaching. Journal of Neurophysiology, 82, 2676-2692.

Neal, A., \& Kilner, J. M. (2010). What is simulated in the action observation network when we observe actions? European Journal of Neuroscience, 32, 1765-1770.

Patel, D., Fleming, S. M., \& Kilner, J. M. (2012). Inferring subjective states through the observation of actions. Proceedings of the Royal Society, Series B, Biological Sciences, 279, 4853-4860.

Pobric, G., \& Hamilton, A. F. (2006). Action understanding requires the left inferior frontal cortex. Current Biology, 16, 524-529.

Press, C., Heyes, C., \& Kilner, J. M. (2011). Learning to understand others' actions. Biology Letters, 7, 457-460.

Ridding, M. C., \& Ziemann, U. (2010). Determinants of the induction of cortical plasticity by non-invasive brain stimulation in healthy subjects. Journal of Physiology, 588, 2291-2304.

Rizzolatti, G., Camarda, R., Fogassi, L., Gentilucci, M., Luppino, G., \& Matelli, M. (1988). Functional organization of inferior area 6 in the macaque monkey. II. Area F5 and the control of distal movements. Experimental Brain Research, 71, 491-507.

Sartori, L., Becchio, C., Bara, B. G., \& Castiello, U. (2009). Does the intention to communicate affect action kinematics? Consciousness and Cognition, 18, 766-772.

Stadler, W., Ott, D. V. M., Springer, A., Schubotz, R. I., Schütz-Bosbach, S., \& Prinz, W. (2012). Repetitive TMS suggests a role of the human dorsal premotor cortex in action prediction. Frontiers in Human Neuroscience, 6, 20.

Tidoni, E., Borgomaneri, S., di Pellegrino, G., \& Avenanti, A. (2013). Action simulation plays a critical role in deceptive action recognition. Journal of Neuroscience, 33, 611-623.

Tkach, D., Reimer, J., \& Hatsopoulos, N. G. (2007). Congruent activity during action and action observation in motor cortex. Journal of Neuroscience, 27, 13241-13250.

Vigneswaran, G., Philipp, R., Lemon, R. N., \& Kraskov, A. (2013). M1 corticospinal mirror neurons and their role in movement suppression during action observation. Current Biology, 23, 236-243. 